\title{
Geografía cotidiana y prácticas culturales: construcción simbólica de los patios del Cementerio General de Santiago
}

\author{
Sebastián Rodríguez Leiva ${ }^{1}$ \\ Camila Salinas Silva²
}

Recibido: 20 de abril de 2015 - Aceptado: 25 de mayo de 2015

\begin{abstract}
Resumen
En este trabajo se analiza la tradición del puesto de cuidador de sepulturas en el Cementerio General de Santiago, un oficio, en muchos casos, heredado familiarmente. Metodológicamente se fundamenta en entrevistas semiestructuradas y mapas mentales, lo cual permitió visualizar la relación existente entre el cuidador y su patio, cargada de simbolismo y vínculos asociados a la temporalidad que posee el puesto y a las prácticas culturales y cotidianas que este desarrolla en su interior. Asimismo, se plantea que el patio se configura como un paisaje de lo simbólico, el que ha representado y representa gran relevancia en la vida del cuidador, como producto del significado y sentido que él ha dotado en este espacio.
\end{abstract}

Palabras clave: Relación simbólica, prácticas culturales, vida cotidiana, cuidador de sepulturas

\section{Daily geography and cultural practices: a symbolic construction of the general cemetery courtyards in Santiago, Chile} Abstract

This work analyzes the tradition of the grave keeper work in the General Cemetery of Santiago, which has been in many cases a familiar inherited job. The methodology is based on semi-structured interviews and mental maps, which allowed visualize the relationship between the gravekeeper and his or her yard, full of symbolism and links associated with the period of time doing that the job and the cultural and daily practices developed. It also considers the yard as a symbolic landscape with great relevance for the life of grave keepers, because all the meaning and sense he or she has given to the place.

Keywords: Symbolic relationship, cultural practices, everyday life, gravekeeper 


\title{
Geografia cotidiana e práticas culturais: construção simbólica dos pátios do cemitério geral de Santiago
}

\begin{abstract}
Resumo
Neste trabalho se analisa a tradição do cargo de zelador de sepulturas no Cemitério Geral de Santiago, um trabalho, em muitos casos, herdado familiarmente. Metodologicamente baseia-se em entrevistas semiestruturadas e mapas mentais, que permitiu visualizar a relação existente entre o cuidador e seu quintal, carregado de simbolismo e vínculos associados a temporalidade que possui o cargo e as práticas culturais e cotidianas que estes desenvolvem no seu interior. Assim também, se manifesta que o pátio, configura-se como uma paisagem do simbólico, que tem representado e representa grande importância na vida dos cuidadores, como produto do significado e sentido que ele tem dado a este espaço.
\end{abstract}

Palavras-chave: relação simbólica, práticas culturais, vida cotidiana, cuidador de sepulturas.

A lo largo de la historia, diversas tradiciones de herencia han estado presentes en la configuración individual, familiar y social de diferentes sociedades y culturas. Durante la época colonial y a lo largo de los siglos XIX y XX en Chile, algunos oficios como el de los artesanos, han sido heredados por parte de los padres a sus hijos de generación en generación, los que con el paso del tiempo se han ido olvidando y perdiendo, como resultado de nuevas experiencias y el escaso interés por compartir y aprender tradiciones, entre otras razones (Mediorural, 2014). Sin embargo, actualmente es posible evidenciar que algunas de ellas aún sobreviven y se mantienen vigentes en el tiempo, como lo es la tradición de heredar el puesto de cuidador de sepulturas y mausoleos en el Cementerio General de Santiago, las que se configuran como relaciones sociales de carácter tradicional que se han perpetuado en el tiempo, transformándose en verdaderas dinastías en el desarrollo de tales prácticas al cuidado de estas sepulturas (Mediorural, 2014).

En este contexto, el presente trabajo pretende dar cuenta de la relación simbólica existente entre el cuidador y el patio que tiene a su cargo en el Cementerio General de Santiago, manifestada en la experiencia propia o heredada y, también, en la integración de discursos y prácticas sociales que tienen una dimensión espacial y cultural explícita y evidente (Damonte, 2011). Con esto, se plantea poder entender y analizar la construcción simbólica de los patios, desde la experiencia y relación del cuidador como figura productora de un espacio dotado de herencia y de tradiciones. Asimismo, se pretende develar aquellos elementos socioculturales que se han desarrollado a lo largo del tiempo, y que dotan de sentido a una espacialidad definida como el patio que tiene a cargo cada uno de los cuidadores de este cementerio. 
Finalmente, cabe decir que la importancia de este trabajo radica en vincular la geografía con la antropología y la sociología, debido principalmente a que esta relación simbólica entre el cuidador y su patio, posee una dimensión geográfica que la convierte en productora de espacio, tanto material y, de forma importante para este trabajo, en espacio simbólico (Mançano, 2000; Giménez, 2001). El trabajo se conforma en cuatro apartados correspondientes en primer lugar a una revisión bibliográfica de los conceptos claves que aborda, el segundo apartado da a conocer de forma breve la metodología empleada, el tercer apartado corresponde al desarrollo del trabajo y, por último, se encuentran las conclusiones y reflexiones finales.

\section{Las nuevas geografías y los paisajes culturales}

A partir de la década de los 80 , en parte como producto del desencanto por las metodologías cuantitativas, la geografía comenzó un proceso importante de reestructuración y renovación en relación a sus contenidos, asumiendo un papel más preponderante la geografía humana, como producto de su apertura hacia las ciencias sociales, y considerando a la identidad como un eje de análisis en la producción del espacio, pasando a analizar los fenómenos a una escala localizada y en un espacio geográfico determinado (Massey et al., 1999; Lindón \& Hiernaux, 2006; Ortega en Romero et al., 2007). De acuerdo a esto, se plantea según Soja (2010) una geografía crítica y enfocada principalmente a los problemas y prácticas sociales bajo una perspectiva postmoderna.

Es en este enfoque conceptual del postmodernismo, que surge la "nueva geografía cultural" consolidada ya en el año 1990 (Nogué, 2007), y que vendría a recalcar ciertos ámbitos que no habían sido tratados anteriormente por parte de la geografía (Claval, 1998). De esta manera, la renovada geografía cultural no sólo se orientaría en los aspectos culturales y descriptivos del espacio, sino también en el espacio visto a través de la óptica de diferentes culturas, lo que permitiría entender de mejor forma los procesos de construcción y producción de éste, siendo considerado como un eje fundamental dentro del enfoque cultural en las ciencias geográficas (Fernández, 2006).

De esta forma, la geografía cultural se abre paso a comprender el espacio como un paisaje cultural, entendiendo, por un lado, que la cultura corresponde a los valores y tradiciones que caracterizan a una sociedad, que han sido transmitidas a través de la historia hasta llegar a la actualidad y que son susceptibles de ser modificados y transmitidos en el futuro (González, 2012). Asimismo, es posible constatar que el término cultura posee dos significados fundamentales, siendo el primero de ellos el de formación del hombre, su mejoramiento y perfeccionamiento, mientras que el segundo significado 
indica el producto de esta formación, correspondiente al conjunto de los modos de vivir y de pensar cultivados y civilizados, a los que se le suele dar también el nombre de civilización.

Por otro lado, el paisaje debe ser entendido tanto una realidad física y, también, como la representación que culturalmente nos hacemos de ella. Es también, la fisonomía externa y visible de una determinada porción de la superficie terrestre y la percepción individual y social que genera; un tangible geográfico y su interpretación intangible. Así, el paisaje se comprende como un escenario natural mediatizado por la cultura, que se configura como el resultado de una transformación colectiva de la naturaleza y como una proyección cultural de una sociedad en un espacio determinado (Nogué, 2007).

De esta forma, se desprende que hablar de paisaje es hablar en el fondo de un paisaje cultural. Tal paisaje cultural, se configura como una porción de la superficie terrestre que ha sido modelada, percibida e interiorizada en un período de tiempo, que pueden ser décadas o siglos, por las sociedades que han vivido y viven en ese entorno. En este contexto, el paisaje está lleno de lugares que encarnan las experiencias y aspiraciones de las sociedades y personas que han estado en él. Tales lugares se convierten en centros de significados que expresan pensamientos, ideas, emociones, tradiciones y costumbres, como producto de una construcción que presenta el mundo tal como es y de una construcción histórica de la sociedad y que permite ver el mundo mediante lo que se configura como un paisaje (Nogué, 2010).

De este modo, el paisaje cultural representa en la superficie terrestre, las diferentes experiencias que los individuos han percibido durante décadas, lo cual guarda estrecha relación con la herencia de tradiciones y costumbres, de generación en generación durante años. Tal herencia de tradiciones y costumbres está dada por un contexto histórico, social y principalmente cultural, las que se llevan a cabo en un tiempo vivido o cotidiano -el ciclo de las 24 horas-, correspondiente al tiempo de las prácticas de los individuos, el que transcurre constantemente y que se configura en lo que se denomina como la vida cotidiana (Lindón, 2000).

\section{La geografía cotidiana y el carácter espacial de las prácticas culturales}

El estudio de la geografía de la vida cotidiana, supone el desafío de construir una mirada geográfica particular, la cual consta de entender por un lado el mundo en el que se inserta esta vida cotidiana y, por otro, la espacialidad que éste posee. Al respecto, Lefebvre (1972) plantea que la vida cotidiana se compone por diferentes elementos que la configuran, los cuales 
corresponden al espacio, el tiempo, las pluralidades de sentido, lo simbólico y las prácticas (Lindón et al., 2006).

Lefebvre (1972) plantea que el espacio es lo que subjetivamente corresponde al entorno de un individuo o un grupo, en el cual se sitúan y viven los individuos. El espacio posee la doble cualidad de presentarse como un lugar que es dotado de sentido por lo sujetos y que, a su vez, le da sentido a los sujetos que interactúan en este lugar. De esta forma, se plantea que el espacio en el cual se desarrolla la vida cotidiana, es donde los actores ejecutan las prácticas que están cargadas de significados y de límites (Lefebvre, 1972).

Por su parte, es imposible concebir lo cotidiano fuera de un contexto de tiempo, en donde se plantea la existencia de un tiempo vivido o tiempo de lo que se denomina como las 24 horas del día, en el cual las prácticas de los individuos transcurren de forma constante. Este tiempo cotidiano, que lleva consigo lo cíclico de la repetición, la evocación y la resurrección, también se inserta en un tiempo cósmico, que proviene de los ritmos de la naturaleza. Simultáneamente, este tiempo cotidiano está dentro de un contexto de tiempo histórico, que a su vez está inserto en un devenir histórico (Lefebvre, 1961).

Respecto a las pluralidades de sentido, Lefebvre (1972) plantea que los diferentes sentidos que están asociados a los puntos de vista que se insertan en la vida cotidiana de los actores, están en relación a un determinado espacio. Por último, las prácticas de la vida cotidiana no sólo corresponden a las prácticas que son ejecutadas por un individuo, sino que corresponden a los encadenamientos y conjuntos que integran a estas prácticas formando un todo, configurándolas en la concepción de lo cotidiano (Lefebvre, 1972).

Asimismo, se debe comprender a la vida cotidiana no sólo como las actividades especializadas de estos ámbitos, sino que también los deseos, las capacidades y posibilidades del ser humano con referencia a todos esos ámbitos, profundizando sus relaciones con los bienes y con los otros; sus ritmos, su tiempo y su espacio y sus conflictos (Lefebvre, 1972: 88). En última instancia, la vida cotidiana para Lefebvre (1972), es la vida del ser humano desplegada en una pluralidad de sentidos y simbolismos, en espacios que lo modelan y al que también dan forma, dentro del flujo incesante de la vivencia del tiempo. Del mismo modo, Lefebvre señala que la vida cotidiana efectuada por cada individuo, no sólo corresponde a las prácticas o acciones que las personas puedan desarrollar, sino que al conjunto de estas prácticas o acciones que se integran formando un todo, tomando en cuenta el contexto en el cual se desarrollan, el sentido que éstas poseen y que las personas le otorgan, ya que dicho conjunto de prácticas se efectúa en un espacio social y en un tiempo social (Lefebvre, 1981: 8). 
Las prácticas y acciones cotidianas que en su conjunto se desarrollan en un espacio social y en un tiempo social, guardan estrecha relación con lo planteado por Lindón (2000: 85), quien argumenta que la vida cotidiana no es un contenido estático en el tiempo, sino que un proceso dinámico y necesariamente histórico. Además, señala que su especificidad no radica en las prácticas reiterativas, sino en los sentidos que estas prácticas representan y en los modos que son representadas. En este contexto, las prácticas cotidianas que desarrolla la figura del cuidador de sepulturas, deben ser comprendidas bajo la lógica expuesta por Lindón (2000), de la relación espacio-temporalidad que éstas poseen y que configuran la vida cotidiana de las personas, en donde la acción práctica y la vivencia intersubjetiva se desarrollan en un "aquí" y en un "ahora" en cada patio del Cementerio General de Santiago.

En síntesis, esto último se relaciona con el carácter temporal e histórico que posee la vida y sus prácticas cotidianas, para lo cual Lefebvre (1972) es muy enfático respecto de tal relación, argumentando que la vida cotidiana no se puede captar en su escala aparente, como se percibe la vida del cuidador de sepulturas en su contexto. En este sentido, el autor plantea que lo micro hay que verlo en una totalidad, es decir, a la luz de la historicidad.

\section{Metodología}

La metodología empleada para llevar a cabo el presente trabajo, se basó principalmente en la ejecución y análisis de entrevistas semiestructuradas y mapas mentales, los que se aplicaron a un total de ocho cuidadores del Cementerio General de Santiago, un hombre y siete mujeres, con edades que fluctuaban entre los 49 y 86 años a noviembre de 2014 y que tenían distintas relaciones de parentesco de herencia familiar. En el texto los hemos identificado tan solo por su nombre con el propósito de resguardar su privacidad. Cada entrevista se enfocó en conocer y analizar la relación simbólica del cuidador con su patio, a partir de las prácticas que desarrolla al interior de este espacio, considerando la tradición histórica-cultural que posee su puesto, la identificación y análisis de las prácticas que desarrolla al interior de su patio y, por último, conocer y comprender la relación que posee el cuidador con su patio, a partir del carácter simbólico que este espacio ha representado y representa en su vida.

\section{Los patios del Cementerio General como un espacio vivido}

A pesar de ser un espacio construido bajo el objetivo de la consagración de la muerte en el siglo XIX, el Cementerio General de Santiago resulta ser un 
espacio que va más allá del sentido religioso y simbólico de una comunidad y de las múltiples concepciones que se construyen de este espacio como un paisaje de la muerte, transformándose en un cobijo de distintas concepciones espaciales y experiencias de vida que configuran a este espacio público, en un espacio simbólico cargado de experiencias de vida, vínculos y simbolismos (Gámez, 2004). El carácter simbólico que posee el patio para el cuidador, se ha ido construyendo a lo largo del tiempo, de generación en generación, a través de la tradición de heredar el puesto de cuidador en el cementerio, lo cual ha sido una expresión de vida tanto para éste, como también para la familia que lo ha acompañado en su vida.

De esta manera, la relación que posee el cuidador con su patio se encuentra dada por múltiples sentimientos que como producto del tiempo y de los años, han configurado una vida al interior de este espacio, provocando que el diario vivir del cuidador esté cargado de una serie de vínculos y simbolismos. Tal relación del cuidador con su patio se ve plasmada en lo planteado por Aínsa (2003), en que el patio pasa a ser un espacio vivido para el cuidador, como producto de la experiencia y del contacto que este ha generado con el lugar, lo que se refleja en el siguiente comentario de una entrevistada: "He trabajado acá toda mi vida, con este trabajo he conocido muchas personas y me siento feliz de poder alimentar y mantener a mi familia, esto ha sido mi vida" (Entrevista a Carmen, 2014).

A partir de esto, se evidencia que el patio es el lugar donde los cuidadores pasan gran parte del día, en donde han crecido y criado a sus hijos, desarrollando un sentido de pertenencia con este espacio, que lo configura en un segundo hogar para el cuidador, otorgándole el carácter de interior a un espacio que es concebido por la sociedad como un espacio de exterior (Aínsa, 2003). En este segundo hogar, el cuidador desarrolla las prácticas cotidianas que normalmente realizaría en su hogar, tal como lo señala otra entrevistada: "Esta es la segunda casa que tengo (...) Yo me doy unas vueltas, me siento y hago mi trabajo, esta es mi vida" (Entrevista a María, 2014).

Este comentario permite dar cuenta de que la relación existente entre el cuidador y su patio como segundo hogar, se basa principalmente en la vinculación de la identidad y el lugar, siendo este último definido por Barros (2000: 82) como "ámbitos de residencia a través de los cuales tanto los individuos en particular y las comunidades, construyen su identidad". En este contexto, la relación que establece el cuidador con su patio, genera un sentido de pertenencia e identidad que podría plantearse como "la expresión de sentirse en casa" (Ver Figura 1) (Lindón et al., 2006: 380). 
Figura 1: Cuidadoras en su cuarto de trabajo

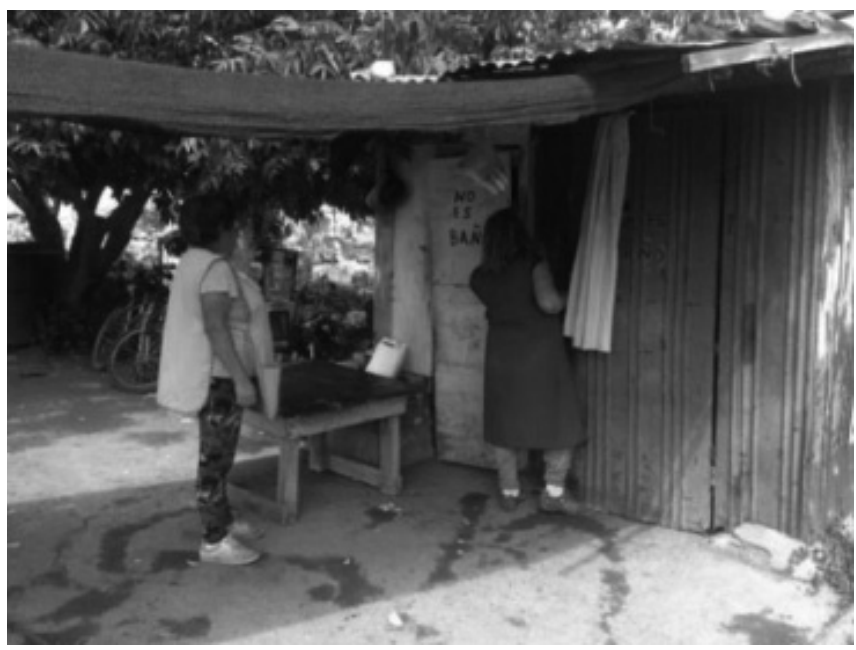

Fuente: Colección propia

Por último, tal es la relevancia que alcanza el patio para el cuidador, que parece ser que la vida fuera de él carece de sentido e importancia. Al respecto, se constata que el cuidador, al estar fuera del patio, piensa constantemente en este espacio y en el cuidado que este necesitaría en su ausencia, tal como se demuestra en la siguiente reflexión "Acá dentro es diferente a cuando uno sale del Cementerio, afuera hay mucho ruido y es estresante" (Entrevista a Alicia, 2014). Tal situación, evidencia el arraigo que posee el cuidador con su patio, en lo que Tuan (2007) plantea como el sentimiento o afectuosidad que posee el hombre con un determinado lugar que le es altamente significativo, otorgándole una serie de calificativos asociados al placer de pertenecer a él.

Sumado a lo anterior, es posible evidenciar que el cuidador experimenta un sentimiento de felicidad hacia su patio, ya que se configura como un espacio en el que puede estar tranquilo, relejarse y llevar una vida placentera, tal como lo plantea un cuidador que dice que "este es un lugar agradable, tranquilo y de paz, puedo dormir a veces en las tardes" (Entrevista a Juan, 2014). Por otro lado, se constata que el cuidador al dejar de trabajar en este puesto y no volver a su patio, pareciera ser que no puede relacionarse con su otra vida, es decir, la vida fuera del Cementerio una vez terminada su jornada laboral, llegando en algunos casos a no poder sobrellevar su propia vida, aislándose en la estrecha relación que posee con su patio.

De esta forma, el cuidador le otorga al patio una existencia real y latente de lo que significa la vida dentro de él, diferenciando al patio del mundo exterior, 
en el que este último se vuelve más negativo, calificándolo de agobiante y estresante, sin descanso y con mucho ruido. Esto se contrapone a las palabras valorativas que el cuidador ofrece para describir la vida al interior del patio, como la paz, la tranquilidad, la seguridad y el bienestar que este espacio le produce al volver a incorporar sus prácticas cotidianas en él.

\section{Construcción simbólica de los patios del Cementerio General}

A partir del análisis de las prácticas espaciales que el cuidador desarrolla en su patio, se evidencia que este espacio redundantemente adquiere las dos escalas que éste posee, mostrando su naturaleza dual compuesta tanto por lo material y, también, de forma importante para este trabajo, lo que denomina como lo simbólico (Oslender, 2010).

El espacio definido por el patio, se encuentra cargado de una serie de sentimientos que han sido otorgados por el cuidador, tal como lo expresa la cuidadora Sonia, quien plantea que la galería que tiene a su cargo es prácticamente su vida (Ver Figura 2), donde ella transita a diario y se moviliza para llegar a los diferentes rincones del espacio que tiene a su cargo, otorgándole gran relevancia e importancia a este lugar. Asimismo, se evidencia que la cuidadora Sonia posee gran arraigo con el cuarto en el cual ella deja sus cosas y descansa cuando tiene tiempo libre (Ver Figura 3). Al respecto, plantea que el cuarto tiene gran significado en su vida, ya que en él puede dialogar consigo misma y sobrellevar los problemas que tiene en su vida, tal como se refleja en el siguiente comentario: "En mi casa tengo una pieza donde guardo mis cosas, donde yo me encierro y hablo sola o con Dios (...) En mi caseta yo puedo hacer todo eso, cuando estoy triste voy ahí un rato" (Entrevista a Sonia, 2014).

Tales vínculos que se van construyendo en el espacio, guardan relación con los planteado por Tuan (2007), quien define esto como la topofilia, es decir, fortaleciendo el conjunto de relaciones emotivas y afectivas, que unen al hombre con un determinado lugar, en donde se desenvuelve aperiódicamente, como es el patio para el cuidador. Asimismo, de acuerdo a lo expuesto por Tuan (2007), se desprende que el cuidador se desenvuelve en una espacialidad explícita que son los patios, en donde mantiene una compleja relación de arraigo y pertenencia, lo que se entrecruza con el sentido de existencia planteado por Arendt (1958), lo que interioriza un vínculo de afecto despertando un sentido reverencial en cada práctica cultural y cotidiana, que el cuidador revive a diario con las distintas experiencias con su lugar de trabajo en el Cementerio General de Santiago. 
Figura 2: Galería del patio de Sonia

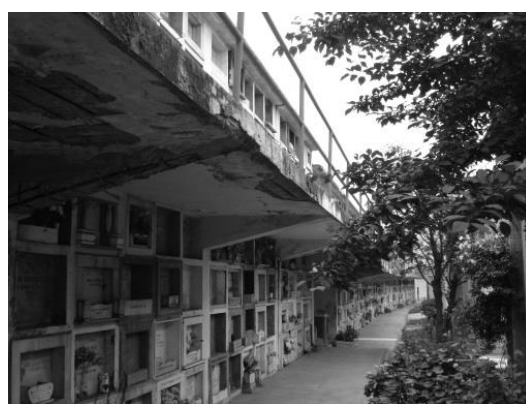

Figura 3: Cuarto de la cuidadora Sonia

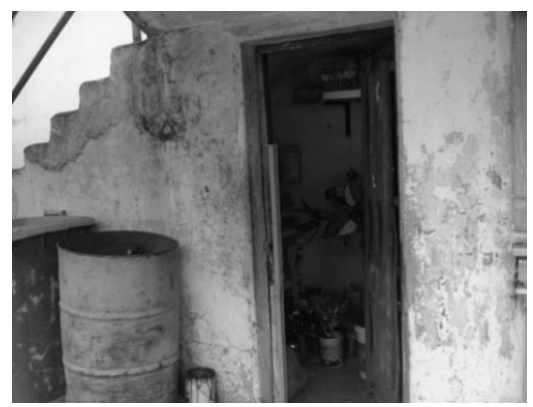

Fuente: Colección propia

El carácter simbólico que posee el patio, está dado por los múltiples elementos que lo configuran y que tienen un significado cotidiano para la vida de cuidador. Esto se constata en la relación que posee la cuidadora Margarita con su patio, donde se observa la existencia de elementos que poseen un carácter simbólico en la conformación de su vida y en lo cotidiano del día a día, como lo es la capilla existente en su patio (Ver Figura 4), la cual posee gran importancia, configurándose en algo central para ella al momento de hablar de sus hijos, ya que fue en este lugar donde ella los dejaba jugando, los protegía y los cuidaba cuando ella trabajaba. Esto se ve reflejado en el siguiente comentario que ella realiza respecto de la capilla: "Yo dejaba jugando a mis hijos acá dentro de la capilla cuando salía a comprar". "Los dueños de la capilla siempre han sido buenos conmigo" (Entrevista a Margarita, 2014).

Figura 4: Capilla presente en el patio de la cuidadora Margarita

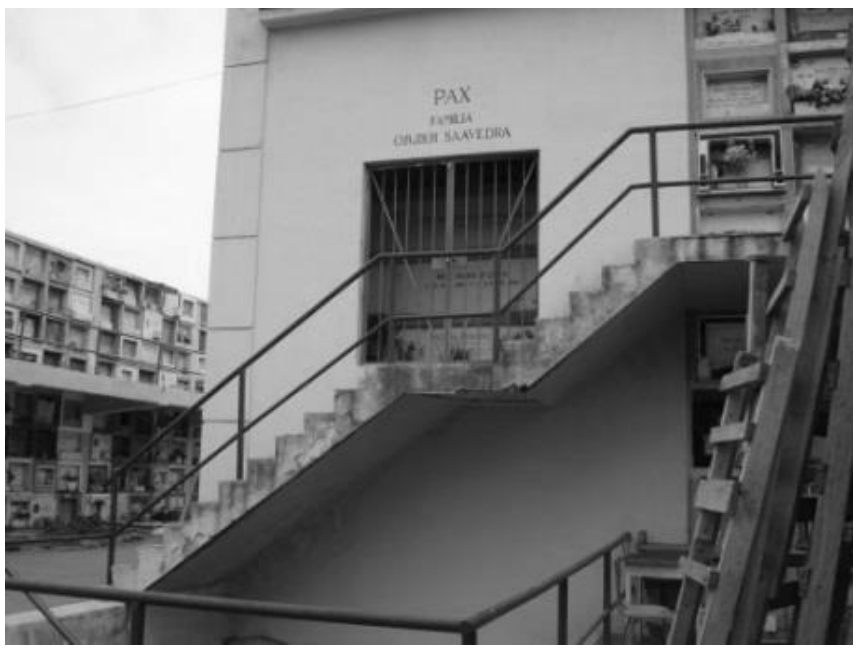

Fuente: Colección propia 
Sumado a lo anterior, se evidencia que la cuidadora Margarita guarda una estrecha relación con las familias que tienen sus sepulturas en el patio que tiene a su cargo, experimentando diferentes sentimientos respecto a la forma en que las personas fallecieron y, de cierta forma, cargando en su vida el dolor que significa la muerte para determinados familiares. Se plantea al respecto, que los sentimientos propios de personas desconocidas como lo son los familiares de los fallecidos de este patio, son también parte de la vida del cuidador y de los sentimientos que este experimenta. Esto se evidencia en que la cuidadora Margarita tiene un sentimiento de pena con el lugar en donde se encuentra la sepultura de un niño que ella llama como Patito (Ver Figura 5), ya que recuerda la forma como él falleció y el dolor que aún sienten sus padres, lo cual se refleja en el siguiente comentario: "Siento pena con los niños que han muerto y que están acá. Es triste saber lo que les pasó" (Entrevista a Margarita, 2014).

Respecto a los elementos propios de su diario vivir, se constata que existen ciertos objetos o materiales que están cargados de significado en la vida de la cuidadora Margarita. Al respecto, existe una mesa en la cual ella puede descansar y sentirse tranquila, la que tiene gran relevancia en su vida cotidiana, en cuanto al desarrollo de sus actividades diarias (Ver Figura 6). Asimismo, se observa que la mesa presenta un sentido de descanso para la vida de la cuidadora, tal como se refleja en el siguiente comentario: "La mesa es el lugar donde almuerzo, donde descanso y donde puedo estar tranquila" (Entrevista a Margarita, 2014).

Figura 5: Sepultura de niño Patito

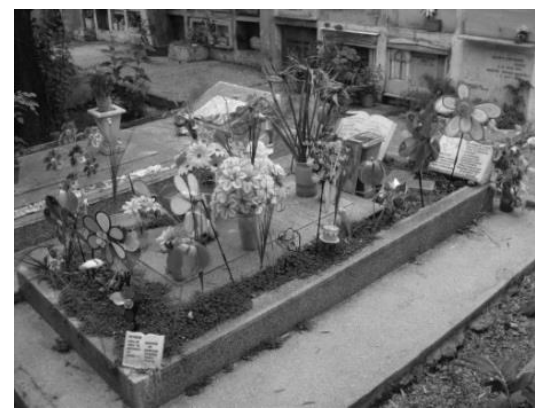

Fuente: Elaboración propia
Figura 6: Mesa de descanso

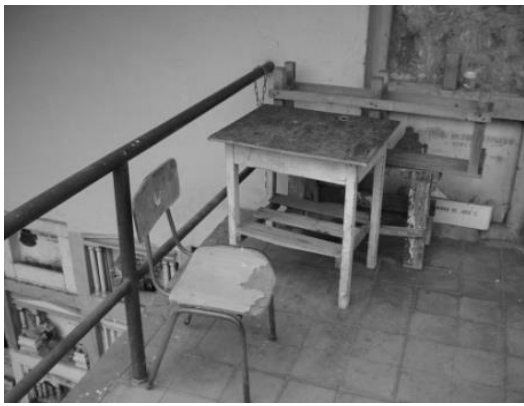

Fuente: Elaboración propia

En definitiva, es posible constatar que el patio se encuentra cargado de elementos, situaciones y experiencias que poseen un carácter simbólico en la vida del cuidador. Esto es producto de la conformación de este espacio a lo largo del tiempo, a través de las experiencias que han acompañado la vida de la cuidadora, en su relación con otras personas, ya sea los familiares que tienen a sus seres queridos en las sepulturas, como también de las re- 
laciones de carácter más personal, como lo son el cuidado de sus hijos y la crianza de estos al interior del patio. Desde esta perspectiva, se entiende el patio como un espacio cargado de múltiples sentimientos, que representa diferentes emociones en la vida de la cuidadora y que hasta lo que podría pensarse como algo irrelevante, como lo es una mesa de madera y una silla mal cuidada, poseen un valor simbólico en la vida del cuidador que sólo es entendible desde la lógica de la importancia que este objeto alcanza en el desarrollo de las prácticas cotidianas existentes al interior del patio.

\section{Conclusiones}

La relación constante entre los cuidadores y el patio que tienen a su cargo está cargada de simbolismo y sentimientos que se han ido construyendo a lo largo del tiempo, como producto de la experiencia individual y la experiencia que ha dejado la espacialidad de la tradición de heredar el puesto de cuidador. Asimismo, es posible evidenciar que esta lógica se construye a partir de la reiteración de afectos y vínculos asociados al placer, la tranquilidad, la seguridad y el arraigo manifestado por los cuidadores en el lugar en el que ellos se desenvuelven: el patio. Tales afectos y vínculos responden a una lógica de poner en valor el espacio que conforma el patio, otorgándole el calificativo de segundo hogar a este espacio, dotándolo de significados e importancia en la vida de estos.

En este mismo sentido, se devela que el símbolo del espacio correspondiente al patio está dado por la figura del cuidador, que es capaz de reconocerlo y ser un elemento connotativo. Sumado a esto, se desprende que el patio debe ser entendido como una producción simbólica, pues es dotado de significado y sentido por parte del cuidador, debido a las relaciones que se generan en su interior, como lo son las prácticas cotidianas, culturales y espaciales. Tal relación, se contrapone o irrumpe con el primer acercamiento que social e históricamente se ha representado y se representa en el Cementerio General de Santiago, como un espacio destinado y dedicado preferentemente a la muerte. Esto representa la dualidad de escalas o doble naturaleza que pueden configurar un paisaje cultural, en el que la vida al interior del patio desarrollada por el cuidador, se configura a una escala de mayor detalle o específica, como también este espacio de la muerte como lo es el Cementerio, se configura a una escala social que posee una mayor generalidad.

\section{Referencias bibliográficas}

Aínsa, F. (2003). “Del Espacio Vivido al Espacio del Texto, Significación Histórica y Literaria del Estar en el Mundo", en Revista CUYO, Anuario de Filosofía Argentina y Americana, 20, pp. 19-36. 
Arendt, H. (1958). La Condición Humana. Traducción de Ramón Gil Novales, Barcelona, España: Editorial Paidós.

Barros, C. (2000). "Reflexiones sobre la Relación entre Lugar y Comunidad", en Revista Documentos de Análisis Geográfico, 37, Barcelona, pp. 81-94.

Benach, N. \& Albet, A. (2010). Edward W. Soja: la perspectiva postmoderna de un geógrafo radical. Barcelona: Editorial Icaria.

Claval, P. (1998). "Los fundamentos actuales de la geografía cultural", en Doc. Anàl. Geogr., 34, pp. 25-40 pp.

Damonte, G. (2011). "Construyendo territorios: narrativas territoriales aymaras contemporáneas". Recuperado el 22 de junio de 2015 en http://biblioteca.clacso. edu.ar/clacso/becas/20120417125730/construyendo.pdf.

Foucault, M. (1984). Espacios Otros. Conferencia Pronunciada en el Círculo de Estudios Arquitectónicos, en 1967. Traducción de Marie Lourdes. Recuperado el 18 de agosto de 2014 en http://148.206.107.15/biblioteca_digital/estadistica.php?ld_host=6\&tipo=ARTICULO\&id=1932\&archivo=7-132-1932qmd. pdf\&titulo=Espacios\%20otros.

Gámez, V. (2004). "Los Espacios de la Muerte como Patrimonio Urbanístico en el Santiago Republicano del siglo XIX", en Revista Electrónica DU\&P. Diseño Urbano y Paisaje, V. I, 1. Centro de Estudios Arquitectónicos, Urbanísticos y del Paisaje de la Universidad Central de Chile.

Giménez, A. (2001). "Cultura, migraciones y aproximaciones teóricas", en Revistas científicas de América Latina, del Caribe, España y Portugal. Universidad Autónoma Metropolitana de Iztapalapa, V. 11, 22, pp. 5-14.

González, A. (2012). Geografía Cultural. Universidad ARCIS. Recuperado el 06 de octubre de 2014 en http://www.buenastareas.com/ensayos/Geograf\%C3\%adaCultural/6902385.html.

Hiernaux-Nicolas, D. (2005) "¿identidades móviles o movilidad sin identidad? El individuo moderno en transformación", en Revista de Geografía Norte Grande, 34, pp. 5-17.

Lefebvre, H. (1961). Crítica de la Vida Cotidiana. Vol II. Recuperado el 08 de octubre de 2014 en http://148.206.107.15/biblioteca_digital/articulos/12-2644134sbt.pdf.

Lefebvre, H. (1972). La Vida Cotidiana en el Mundo Moderno. Madrid: Alianza Editorial. Recuperado el 30 de agosto de 2014 en http://148.206.107.15/biblioteca_digital/articulos/12-264-4134sbt.pdf.

Lefebvre, H. (1974). "La Producción del Espacio", Revista de Sociología, 3, pp. 219-229. 
Lefebvre, H. (1981). Crítica de la Vida Cotidiana. Vol III. Recuperado el 08 de octubre de 2014 en http://148.206.107.15/biblioteca_digital/articulos/12-2644134sbt.pdf.

Lindón, A. (2000). La Vida Cotidiana y su Espacio-Temporalidad. Barcelona: Editorial Anthropos-CRIM-El Colegio Mexiquense. Recuperado el 30 de agosto de 2014 en http://www.ub.edu/geocrit/b3w-380.htm.

Lindón, A., Hiernaux, D., Bertrand, G. (2006). Tratado de Geografía Humana, Barcelona, España, Editorial Anthropos.

Mançano, B. (2000). "Movimientos socioterritoriales y movimientos socioespaciales. Contribución teórica para una lectura geográfica de los movimientos sociales". Recuperado el día 01 de septiembre, en: http://web.ua.es/en/giecryal/ documentos/documentos839/docs/bmfunesp-5.pdf.

Massey, D., Allen, J. y Sarre, P. (Eds.) (1999). Human geography today. Cambridge: Polity Press.

Mediorural (2014). "La Dinastía de los Cuidadores de Cementerios". Recuperado el 30 de agosto de 2014 desde: http://www.mediorural.cl/index.php/el-mito/171la-dinastia-de-los-cuidadores-de-cementerios.

Nogué, J. (2007). "Paisaje, Identidad y Globalización". Recuperado el 13 de octubre de 2014 en http://www.ehu.es/ojs/index.php/Fabrikart/article/view/2227/1843.

Nogué, J. (2010). "El Retorno al Paisaje". Recuperado el 13 de octubre de 2014 en http://issuu.com/thedafallen5/docs/el_retorno_al_paisaje.

Oslender, U. (2000). "La Búsqueda de un Contra-Espacio: ¿hacia territorialidades alternativas o cooptación por el poder dominante?", en Geopolítica(s): Revista de estudios sobre espacio y poder, V. 1, 1, pp. 95-114.

Romero, J. (2007). Geografía Humana. Procesos, riesgos e incertidumbres en un mundo globalizado. Barcelona: Ariel.

Tuan, Yi-Fu (2007). Topofilia: Un Estudio de las Percepciones, Actitudes y Valores sobre el Entorno. Madrid: Editorial Melusina. 